\title{
Obtenção de Membranas Microporosas a partir de Nanocompósitos de Poliamida 6/Argila Nacional. Parte 1: Influência da Presença da Argila na Morfologia das Membranas
}

\author{
Amanda M. D. Leite \\ Programa de Ciência e Engenharia de Materiais, UFCG \\ Edcleide M. Araújo, Hélio de L. Lira \\ Departamento de Engenharia de Materiais, UFCG \\ Renata Barbosa \\ Universidade Federal do Piauí, UFPI \\ Edson N. Ito \\ DEMat, UFRN
}

\begin{abstract}
Resumo: Membranas poliméricas foram produzidas a partir de nanocompósitos de poliamida 6 e argila constituída de silicatos em camadas, utilizando a técnica de imersão-precipitação. A argila foi modificada organicamente com os sais quaternários de amônio, Dodigen e Cetremide. Foram obtidos nanocompósitos de poliamida $6 \mathrm{com}$ argila sem tratamento (MMT) e com argila tratada (OMMT). Os nanocompósitos obtidos foram avaliados por DRX e MET, apresentando estrutura com predominância de lamelas de argila esfoliadas na matriz polimérica. As membranas produzidas pelo método de inversão de fases foram caracterizadas por DRX e MEV. A difração de raios X das membranas confirmou os resultados para os nanocompósitos anteriormente preparados. A superfície da matriz observada por MEV apresentou poros irregulares. Já para as membranas com os nanocompósitos observou-se maior quantidade e melhor distribuição dos poros, indicando que a presença da argila alterou a morfologia da membrana. As fotomicrografias das seções transversais dessas membranas mostraram uma estrutura morfológica assimétrica, constituída de uma pele, onde os poros são muito pequenos ou inexistentes, e uma camada porosa com poros de tamanho e distribuição uniformes.
\end{abstract}

Palavras-chave: Poliamida 6, nanocompósitos, membranas, inversão de fases.

\section{Obtaining Microporous Membranes from Nylon 6/National Clay Nanocomposites. Part 1: Influence of Clay on the Membranes Morphology}

\begin{abstract}
Polymeric membranes were produced from nylon 6 nanocomposites and a clay using the immersion-precipitation technique. The clay was organically modified by using a quaternary ammonium salt, Dodigen. Nanocomposites were obtained from nylon 6 with untreated clay (MMT) and treated clay (OMMT). The nanocomposites were studied by XRD and TEM. The morphological structure consisted of an exfoliated and partially exfoliated clay layers in the polymeric matrix. The membranes were produced by phase inversion method and characterized by XRD and SEM. The X-ray diffraction of the membranes confirmed the results for the nanocomposites. The SEM image of the membrane top surface showed irregular pores. As for the membranes with the nanocomposites, a larger number of better distributed pores were observed, indicating that the presence of the clay altered the membrane morphology. The cross section SEM image showed an asymmetric morphology structure, comprising two layers, namely a skin with small and closed pores, and a porous layer with large, uniformly distributed pores.
\end{abstract}

Keywords: Nylon 6, nanocomposites, membranes, inversion phase method.

Autor para correspondência: Amanda M. D. Leite, Programa de Ciência e Engenharia de Materiais, UFCG, Av. Aprígio Veloso 882, CEP: 58429-900, Campina Grande, PB, Brasil. E-mail: amandamelissa.lins@yahoo.com.br 


\section{Introdução}

Os processos de separação por membranas, embora recentes, tem sido utilizados de maneira crescente como processos de separação, purificação, fracionamento e concentração numa ampla variedade de indústrias, tais como as químicas, farmacêuticas, têxteis, de papel e alimentícias ${ }^{[1]}$. Estes processos apresentam como principais atrativos, em relação aos processos convencionais de separação, o baixo consumo de ene rgia, a redução do numero de etapas em um processamento, maior eficiência na separação e alta qualidade do produto final ${ }^{[2]}$. O processo de separação por membranas vem empregando membranas poliméricas na técnica de separação de massa e que vem sendo aplicado em vários processos tecnológicos ${ }^{[3]}$.

Atualmente, as membranas mais utilizadas mundialmente são as chamadas de segunda geração, produzidas a partir de polímeros sintéticos como as poliamidas, polissulfonas, poliacrilonotrila, policarbonatos, polieterimida, entre outros. Essas membranas apresentam não só melhor resistência química como também resistência térmica. Possuem boa resistência a compostos clorados, apesar de apresentarem baixa resistência à compactação mecânica e, ainda, estas membranas podem ser utilizadas com solventes não$\operatorname{aquosos}^{[4]}$.

Os nanocompósitos são materiais que compreendem uma dispersão de partículas nanométricas em uma matriz, onde esta pode ser única ou blenda polimérica. Em virtude do pequeno tamanho, as nanopartículas são invisíveis a olho nu, consequientemente podem ser usadas para produzir compostos reforçados, porém transparentes. Os nanocompósitos poliméricos são uma nova classe de materiais que estão emergindo na indústria. Desde o desenvolvimento de nanocompósitos formados por poliamidas e argila, pelo grupo Toyota, os nanocompósitos poliméricos com silicatos laminados têm recebido uma atenção considerável em diversos setores como: laboratórios acadêmicos, industriais e governamentais e, atualmente são estudados por inúmeros grupos de pesquisa em todo o mundo ${ }^{[5,6]}$. Atualmente, vem sendo dada muita atenção aos nanocompósitos poliméricos, especialmente aos desenvolvidos com silicatos em camadas, devido a grande necessidade de materiais modernos de engenharia e ao fato dos polímeros puros não apresentarem as propriedades necessárias para certas aplicações ${ }^{[7]}$.

As poliamidas apresentam importância significativa como um material de desempenho elevado e, ainda, excelentes propriedadesmecânicase térmicas ${ }^{[8]}$.Alémdisso, as poliamidas vêm sendo utilizadas em matrizes de nanocompósitos, onde têm apresentado propriedades interessantes tais como: propriedades de barreira, propriedades térmicas entre outras. Os filmes de nanocompósitos poliméricos baseiam- se na impermeabilização das lamelas de montmorilonitas e recebem considerável atenção desde que conduzam a propriedades de barreira com menor índice de carga do que os filmes convencionais de compósitos ${ }^{[9-12]}$. Este trabalho tem como objetivo obter membranas de nanocompósitos de poliamida 6/argila nacional por meio do método de inversão de fases e avaliar o efeito da argila na morfologia das membranas por: DRX, MET e MEV. O método de inversão de fases consiste de três etapas principais: preparo de uma solução polimérica homogênea, espalhamento da solução sobre uma superfície formando um filme de espessura determinada e, finalmente, formação da estrutura polimérica da membrana pela separação de fases do sistema.

\section{Experimental}

\section{Material}

Argila Bentonita Brasgel PA (sódica), CTC $=90 \mathrm{meq} / 100 \mathrm{~g}$ (método de adsorção de azul de metileno), passada em peneira ABNT n ${ }^{\circ} .200(\mathrm{D}=0,074 \mathrm{~mm})$, de cor creme, fornecida pela Bentonit União Nordeste (BUN), localizada na cidade de Campina Grande - PB. Esta argila foi nomeada de argila sem tratamento (MMT), devido ao argilomineral predominante ser a montmorilonita . Para torná-la compatível com a matriz polimérica, os íons de sódio presentes entre as lamelas da argila são trocados por íons de amônio para produzir a argila organofílica. Essa troca foi realizada na presença dos sais quaternários de amônio Dodigen (cloreto de alquil dimetil benzil amônio), fornecido pela Clariant/PE e Cetremide (brometo de hexadeciltrimetil amônio), fornecido pela Vetec/SP. Para a obtenção da argila organofílica (OMMT) foi realizado tratamento apropriado para o sal, baseandose na CTC da argila, conforme trabalhos anteriormente reportados ${ }^{[13-17]}$.

A matriz polimérica empregada foi a poliamida $6 \mathrm{com}$ massa molar de 10.500 g. $\mathrm{mol}^{-1}$, IV $=134 \mathrm{~mL} \cdot \mathrm{g}^{-1}$ (Technyl C216) fornecida pela Rhodia/SP, sob a forma de grânulos de coloração branca.

Para a preparação das membranas foi utilizado o solvente Ácido Fórmico da Synth, com 99\% de pureza.

\section{Métodos}

\section{Preparação dos nanocompósitos}

Para a produção dos nanocompósitos de poliamida foi preparado um concentrado (1:1) em misturador interno acoplado ao Reômetro de Torque System 90 da Haake-Büchler, operando a $240{ }^{\circ} \mathrm{C}, 60 \mathrm{rpm}$ por 10 minutos. O concentrado obtido foi triturado em moinho de facas e, posteriormente, adicionado à matriz polimérica, em quantidades necessárias para a obtenção de teores nominais de 3\% em massa de argila. A mistura foi processada em extrusora de rosca dupla contrarotativa acoplada a um Reômetro de Torque System 90 da Haake-Büchler, utilizando-se temperatura de $230{ }^{\circ} \mathrm{C}$ na $1^{\mathrm{a}}$ zona e $240{ }^{\circ} \mathrm{C}$ nas demais zonas de aquecimento e velocidade de rotação das roscas de $60 \mathrm{rpm}$. Para efeito de comparação, a poliamida pura foi extrudada sob as mesmas condições da mistura. 


\section{Preparação das membranas}

Para a preparação das membranas foi utilizado o método de inversão de fases, por meio da técnica de imersãoprecipitação. A poliamida 6 e os nanocompósitos (secados a $80{ }^{\circ} \mathrm{C}$ sob vácuo, por um período de 24 horas) foram dissolvidos numa quantidade conhecida de ácido fórmico (na proporção de $20 \%$ em peso de polímero e $80 \%$ em peso de ácido fórmico), a uma temperatura de $40{ }^{\circ} \mathrm{C}$, até a total dissolução do polímero. A poliamida 6 dissolvida em ácido fórmico forma uma solução clara e homogênea, enquanto que, os nanocompósitos formam uma solução turva. Após o preparo da solução, esta foi espalhada em uma placa de vidro e depois imersa rapidamente em um banho de um não-solvente, no caso, água destilada. Depois de concluída a precipitação, a membrana foi removida e lavada com água destilada e secada em estufa a $50{ }^{\circ} \mathrm{C}$ por um período de 2 horas.

\section{Caracterizações}

Os nanocompósitos e as membranas foram caracterizados por DRX, para que fosse determinado o espaçamento basal entre as camadas de argila, bem como verificar a formação do nanocompósito. As análises de DRX foram realizadas em aparelho XRD-6000 Shimadzu, utilizando-se radiação $\mathrm{K} \alpha$ do cobre $(\lambda=1,542 \AA)$, tensão de $40 \mathrm{kV}$, corrente de $30 \mathrm{~mA}$, varredura entre $2 \theta$ de 2 a $30^{\circ}$ e velocidade de varredura de $2 \%$ min, no Laboratório de Engenharia de Materiais/CCT/ UFCG, no pó das argilas sem tratamento e da organofílica, nos nanocompósitos e nas membranas.

Os nanocompósitos foram caracterizados também por microscopia eletrônica de transmissão (MET) que permite analisar a morfologia do nanocompósito. As análises foram realizadas utilizando-se o microscópio eletrônico de transmissão da marca PHILIPS CM120, operando a uma voltagem de aceleração de $120 \mathrm{kV}$, pertencente ao DEMA/UFSCar. As análises foram realizadas em filmes obtidos a partir dos grânulos dos nanocompósitos. As amostras foram preparadas através da redução de área pelo procedimento de "trimmer", em forma trapezoidal com uma área de aproximadamente $0,5 \mathrm{~mm}^{2}$. Os cortes das amostras foram realizados em um ultramicrótomo da marca RMC modelo MT-7000 usando-se uma faca de diamante da marca Diatome tipo Cryohisto $45^{\circ}$, em condições criogênicas, com velocidade de corte de $0,1 \mathrm{~mm} / \mathrm{s}$ e espessura entre 25 a $50 \mathrm{~nm}$.

As membranas foram caracterizadas também por microscopia eletrônica de varredura (MEV) para que fosse avaliada sua morfologia. Essas análises foram obtidas no equipamento SSX 550 Superscan - Shimadzu (CTGÁS-RN). Foram avaliadas a superfície de topo e a seção transversal das membranas. Para análise da seção transversal, as amostras foram fraturadas em nitrogênio líquido para assim evitar sua deformação plástica. As amostras foram recobertas com ouro ("sputtering"-Metalizador Shimadzu-IC-50, utilizando uma corrente de $4 \mathrm{~mA}$ por um período de 3 minutos). O tamanho médio dos poros das membranas foi calculado a partir das imagens de MEV das superfícies de topo das membranas, usando o programa Mocha da Jandell (analisador de imagens), pela contagem média de 180 poros/amostra.

\section{Resultados e Discussão}

\section{Caracterização da argila e do nanocompósito}

\section{Difração de raios X (DRX)}

A Figura 1a, b mostram os difratogramas de DRX da argila sem tratamento (MMT), da argila organofílica (OMMT) e dos nanocompósitos obtidos. Observa-se que

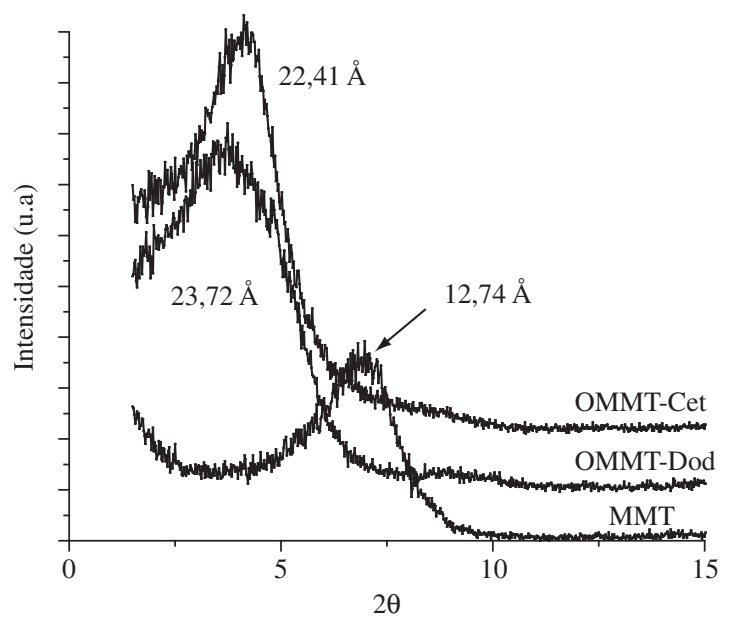

(a)

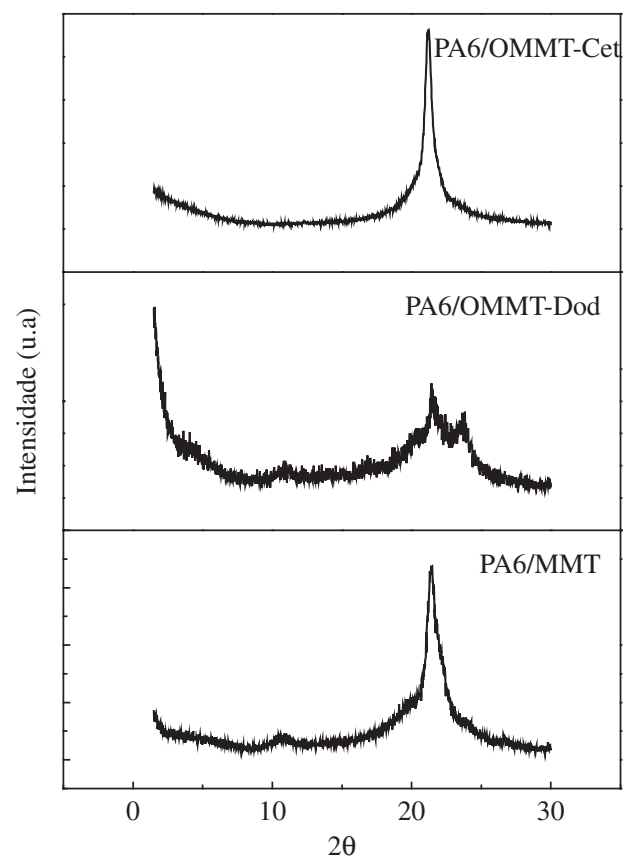

(b)

Figura 1. a) Difratogramas da argila sem tratamento (MMT), da argila tratada com os sais Dodigen (OMMT-Dod) e Cetremide (OMMT-Cet); e b) Difratogramas dos nanocompósitos de PA6/argila. 
houve um deslocamento do ângulo de $2 \theta$ de 6,97 $(\mathrm{MMT}$, $\mathrm{d}_{001}=12,74 \AA$ ) para $3,74^{\circ}\left(\right.$ OMMT-Dod, $\mathrm{d}_{001}=23,72 \AA$ ) e para $4,15^{\circ}$ (OMMT-Cet, $\mathrm{d}_{001}=21,41 \AA$ ). O deslocamento para ângulos menores e, consequentemente, aumento da distância basal, indica que houve a intercalação das moléculas do sal entre as camadas de argila. Para o nanocompósito obtido de poliamida 6 , foi verificada a ausência do pico característico da argila quando a mesma está incorporada na matriz polimérica, podendo assim indicar uma estrutura esfoliada do nanocompósito, o que será confirmado por MET. Esses resultados estão de acordo com a literatura e, aparentemente, revelam que os nanocompósitos apresentam estrutura esfoliada $^{[18-20]}$

\section{Microscopia eletrônica de transmissão (MET)}

As Figuras 2a, b, c ilustram as fotomicrografias dos sistemas de poliamida 6 com $3 \%$ em peso de argila. A fotomicrografia do sistema PA6/MMT apresenta uma morfologia composta de aglomerados dispersos na matriz polimérica, indicados por setas e algumas lamelas de argila, tipo tactóides (aglomerados de uma ou mais lamelas). Já a

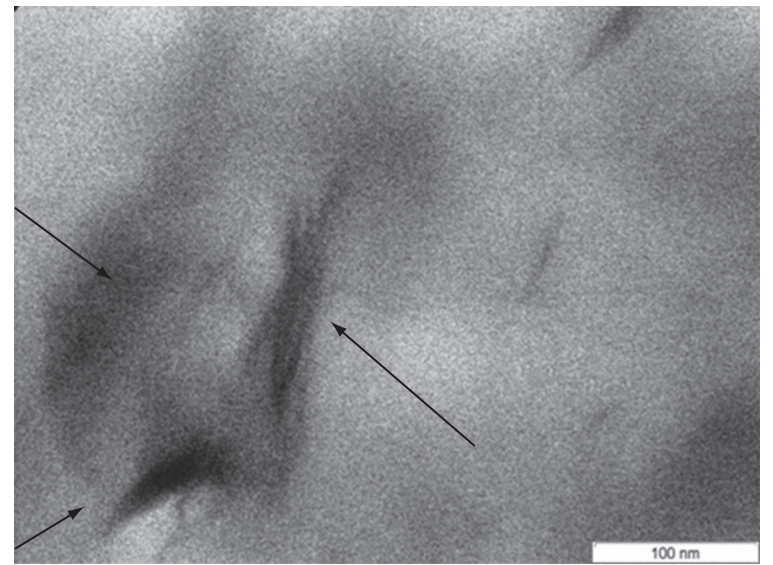

Aumento de $110000 \times$ fotomicrografia do sistema PA6/OMMT-Dod (Figura 2b) mostra uma morfologia esfoliada com presença de lamelas de argila distribuídas de maneira uniforme, apresentando algum aglomerado. Dessa forma, pode-se confirmar que a argila foi organofilizada com o sal quaternário de amônio Dodigen e que nanocompósitos foram obtidos com a argila organofílica e apresentaram uma estrutura com predominância de esfoliação. E a fotomicrografia do sistema PA6/OMMT-Cet apresenta uma morfologia parcialmente esfoliada, composta e lamelas de argilas e alguns aglomerados dispersos na matriz polimérica, sendo que o sistema PA6/MMT apresenta aglomerados de tamanhos maiores.

\section{Caracterização das membranas}

\section{Difração de raios X (DRX)}

A Figura 3 apresenta os difratogramas obtidos por DRX das membranas preparadas a partir da poliamida 6 e seus nanocompósitos. Como nos nanocompósitos, pode-se enfatizar aqui o desaparecimento também do pico característico da argila, confirmando a esfoliação desta nas

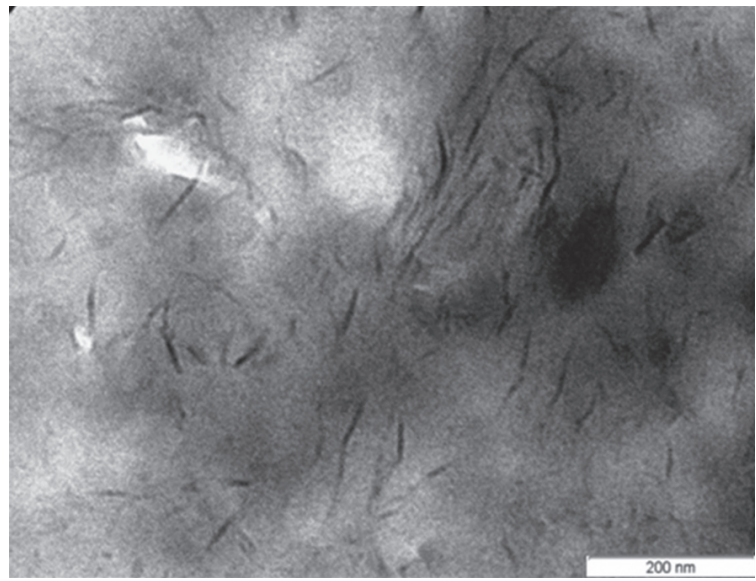

Aumento de 110000x

(b)

(a)

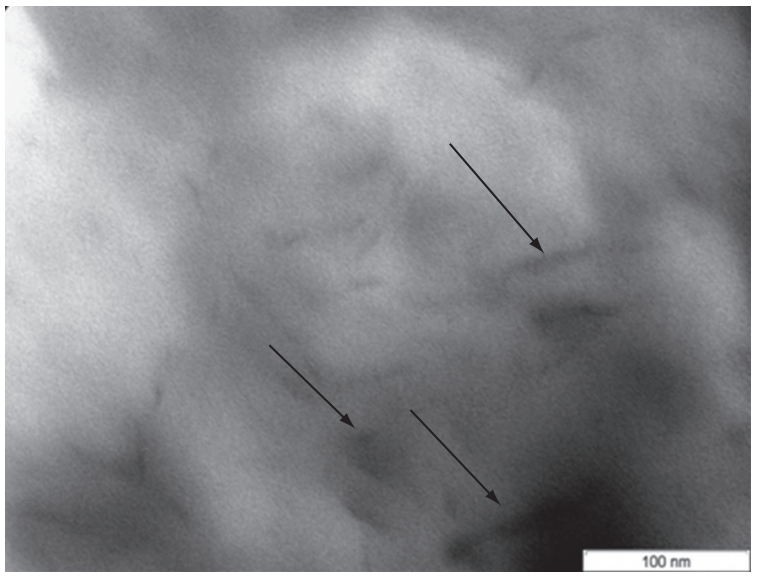

Aumento de 110000x

(c)

Figura 2. Fotomicrografias de MET para os filmes de nanocompósitos de a) PA6/MMT; b) PA6/OMMT-Dod; e c) PA6/OMMT-Cet. 


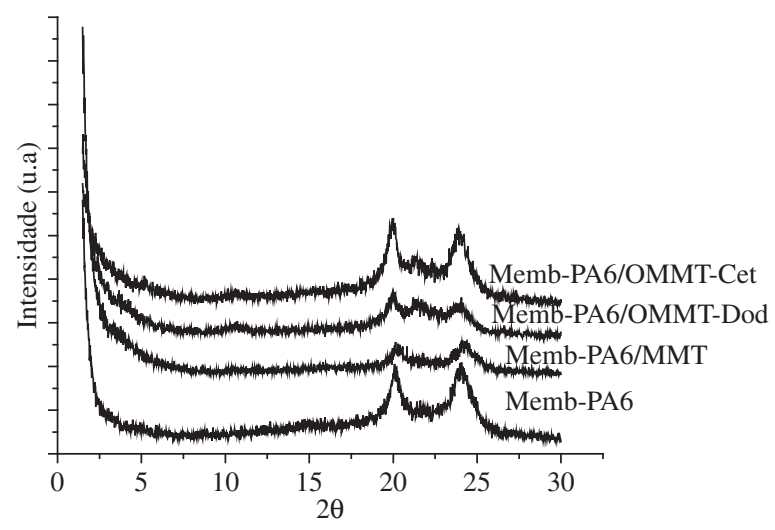

Figura 3. Difratogramas das membranas de poliamida 6 e das membranas obtidas a partir de seus nanocompósitos.

membranas. Percebe-se ainda a presença de dois picos em $2 \theta$ de aproximadamente 20 e $24^{\circ}$, que são mais intensos na membrana de PA6. O aparecimento desses picos pode estar relacionado à formação de uma fase cristalina da poliamida, denominada fase $\alpha$ (alfa). Aparentemente, com a obtenção das membranas, eles tendem a desaparecer, provavelmente devido à perda de cristalinidade dessas membranas ${ }^{[21]}$.

\section{Microscopia eletrônica de varredura (MEV)}

\section{Superfície de topo}

As fotomicrografias de topo das membranas estão apresentadas na Figura 4. Analisando as imagens, podese verificar que as membranas obtidas a partir dos nanocompósitos apresentam uma maior quantidade de poros e distribuição desses de maneira mais uniforme quando comparado à membrana de PA6. Observa-se também que a presença da argila tratada diminui o tamanho dos poros. A membrana de PA6 pura apresenta um tamanho de poro de aproximadamente $0,18 \mu \mathrm{m}$ não - uniformemente distribuídos, enquanto que a membrana de PA6/OMMT-Dod apresenta um tamanho de poro de aproximadamente $0,16 \mu \mathrm{m}$, uniformemente distribuídos, porém para membrana de PA6/OMMT-Cet verifica-se um aumento no tamanho e melhor distribuição dos poros.

Foi verificada ainda por MEV, a presença de algumas partículas na superfície da membrana de PA6. Através da técnica de espectroscopia por energia dispersiva (EDS), acessório do MEV, feita na partícula, foi indicado a presença de elementos característicos da matriz polimérica. A presença desta partícula pode ser atribuída a uma precipitação diferenciada na superfície da membrana ou a uma dissolução incompleta do polímero durante o preparo da solução para a obtenção das membranas.

\section{Seção transversal}

As Figuras 5, 6, 7 e 8 apresentam as fotomicrografias obtidas por MEV da seção transversal das membranas. Nessas fotomicrografias foi possível visualizar uma variação no tamanho dos poros ao longo da espessura da membrana.

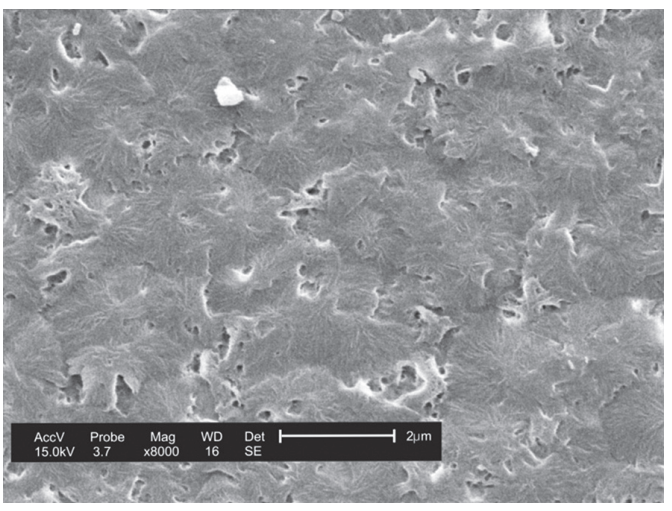

(a)

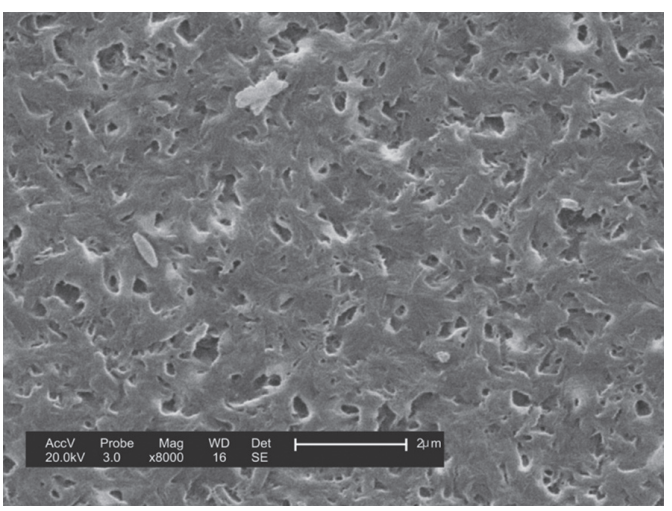

(b)

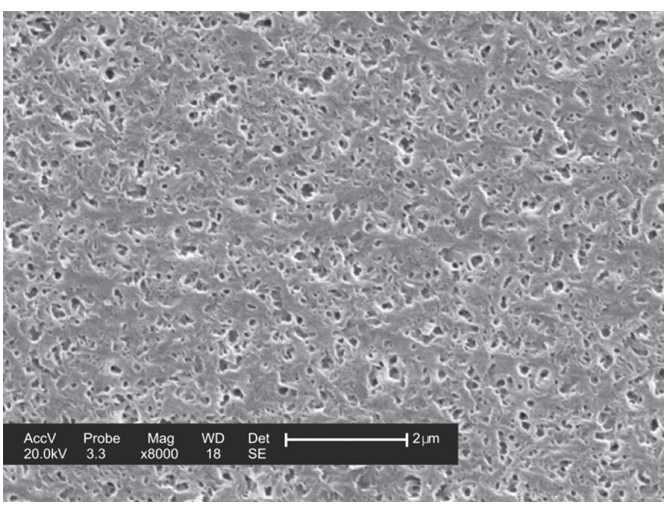

(c)

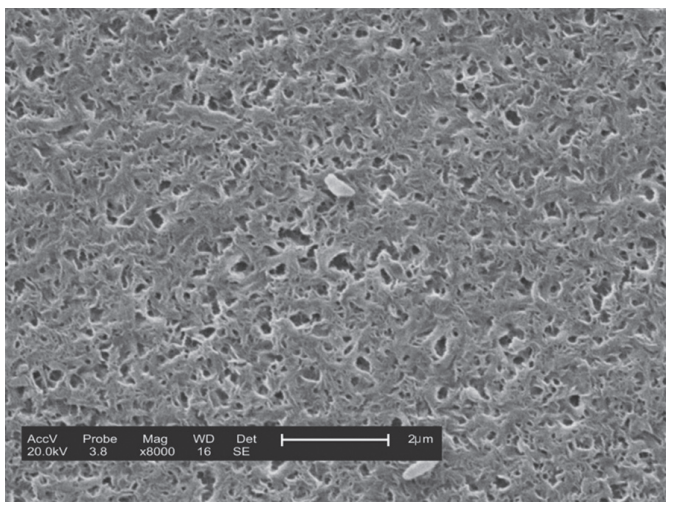

(d)

Figura 4. Fotomicrografias obtidas por $\mathrm{MEV}$ da superfície de topo da membrana de a) PA6; b) PA6/MMT; c) PA6/OMMT-Dod; e d) PA6/OMMT-Cet. 


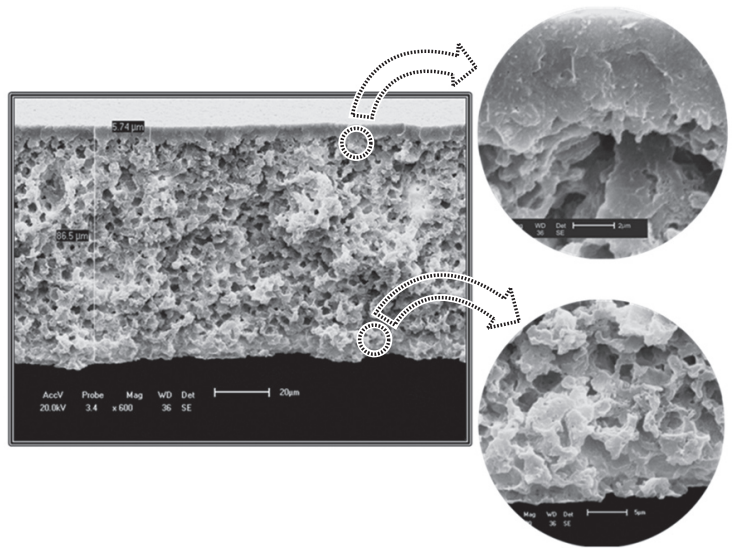

Figura 5. Fotomicrografia obtida por MEV da seção transversal da membrana de PA6.

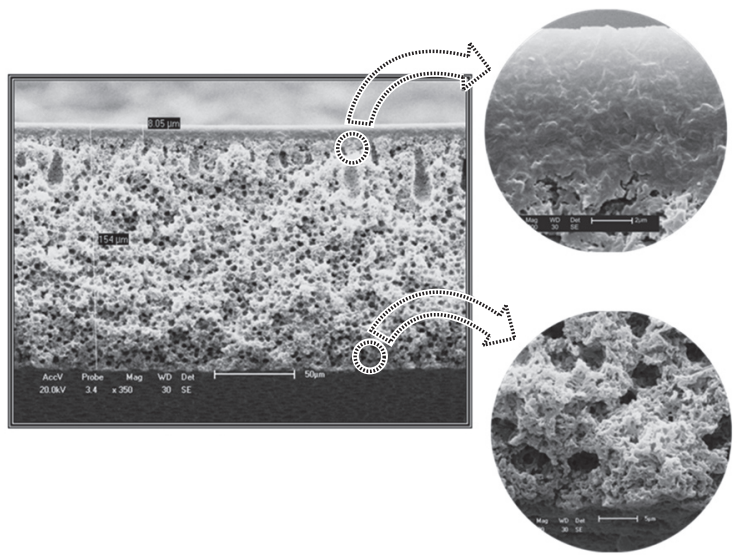

Figura 6. Fotomicrografia obtida por MEV da seção transversal da membrana de PA6/MMT.

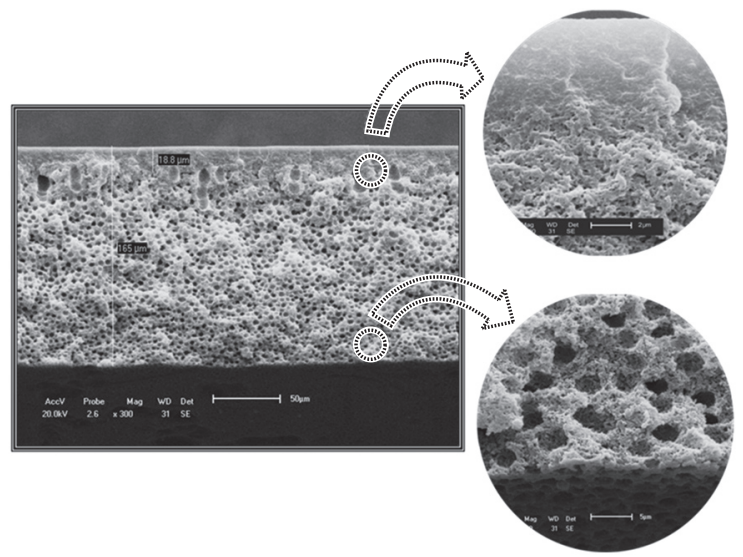

Figura 7. Fotomicrografia obtida por MEV da seção transversal da membrana de PA6/OMMT-Dod.

Para todas elas, verifica-se uma camada denominada de pele filtrante e, logo abaixo a essa pele, a camada porosa, com poros distribuídos de modo uniforme e de forma esférica.

Foi possível fazeruma ampliação nas regiões anteriormente descritas para uma melhor avaliação da morfologia e estas figuras ampliadas mostram a pele filtrante com poros muito pequenos ou até mesmo inexistentes, e a camada porosa com poros interconectados e bem distribuídos. Em todas

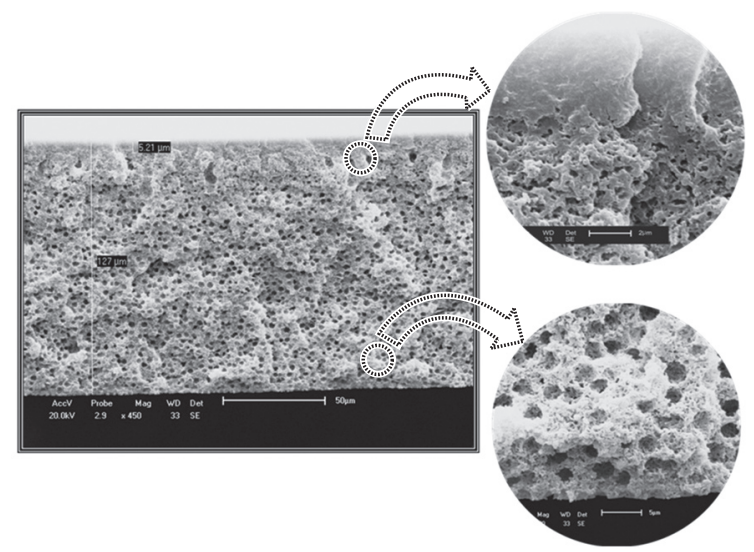

Figura 8. Fotomicrografia obtida por $\mathrm{MEV}$ da seção transversal da membrana de PA6/OMMT-Cet.

as seções transversais analisadas, observou-se a formação de macrovazios e a presença de uma camada densa. Isso provavelmente ocorreu em virtude de uma precipitação com atraso na obtenção da membrana. Dessa diferença de morfologia na seção transversal é que surge ou se define (também) a seletividade da membrana, a qual será investigada em trabalhos futuros através das medidas de fluxo.

\section{Conclusões}

Membranas microporosas assimétricas foram obtidas a partir de nanocompósitos de poliamida 6 com argila, onde a presença da argila proporcionou uma modificação estrutural nas mesmas. Por meio de DRX e MET foram confirmadas predominância de estruturas esfoliadas, sendo visto por MET as lamelas de argila e alguns aglomerados dispersos na matriz polimérica, ou seja, um nanocompósito foi produzido. Os difratogramas de DRX das membranas comprovaram a estrutura esfoliada e/ou parcialmente esfoliadas dos nanocompósitos. As fotomicrografias de MEV das membranas obtidas a partir dos nanocompósitos apresentaram uma maior quantidade de poros e uma melhor distribuição dos poros quando comparadas à membrana de poliamida 6 .

\section{Agradecimentos}

Os autores agradecem a Bentonit União Nordeste (BUN) pelo fornecimento da argila, à Rhodia pela doação da Poliamida 6, ao Prof. Dr. Elias Hage Jr. (DEMa/UFSCar) pela realização dos ensaios de microscopia, à RENAMI (Rede de Nanotecnologia Molecular e de Interfaces), à ANP/PRH-25, LABMat - Laboratório de Eng. de Materiais/CCT/UFCG, ao DEMa/UFSCar, ao CTPETRO/MCT/CNPq e MCT/CNPq pelo apoio financeiro.

\section{Referências Bibliográficas}

1. Hamza, A.; Pham, V. A.; Matsuura, T. \& Santerre, J. P. J. Membr. Sci., 131, p.217 (1997). 
2. Petrus, J. C. C. - "Preparação, modificação $e$ caracterização de membranas assimétricas para clarificação de suco de frutas", Tese de Doutorado, Universidade Estadual de Campinas, São Paulo, Brasil (1997).

3. Sysel, P.; Sindelar, V.; Kubonova, M.; Friess, K.; Hynek, V. \& Sipek, M. - Desalination, 236, p.46 (2009).

4. Basseti, F. J. - "Preparação, caracterização e aplicação de membranas poliméricas microporosas assimétricas", Tese de Doutorado, Universidade Estadual de Campinas, São Paulo, Brasil (2002).

5. Sinha Ray, S. \& Okamoto, M. - Poly. Sci., 28, p.1539 (2003).

6. Pinnavaia, T. J. \& Beall, G. W. - "Polymer-Clay Nanocomposites", John Wiley \& Sons, Toronto (2000).

7. Paz, R. A.; Leite, A. M. D.; Araujo, E. M.; Melo, T. J. A.; Barbosa, R. \& Ito, E. N. - Polímeros: Ciência e Tecnologia, 18, p.341 (2008).

8. Espeso, J.; Lozano, A. E.; Campa, J. G. \& Abajo, J. - J. Membr. Sci., 280, p.659 (2006).

9. Messersmith, P. B. \& Giannelis, E. P. - J. Polym. Sci., 33, p.1047 (1995).

10. Chang, J. H.; An, Y. U. \& Sur, G. S. - J. Polym. Sci., 41, p.94 (2003).

11. Gain, O.; Espuche, E.; Pollet, E.; Alexandre, M. \& Dubois, P. H. - J. Polym. Sci., 43, 2, p.205 (2005).

12. Ranade, A.; D'Souza, N. A.; Gnade, B.; Dharia, A. - J. Plastic Film Sheeting, 19, p.271 (2003).
13. Barbosa, R.; Araújo, E. M.; Oliveira, A. D. \& Melo, T. J. A. - Revista Cerâmica, 52, p.264 (2006).

14. Araújo, E. M.; Melo, T. J. A.; Santana, L. N. L.; Neves, G. A.; Ferreira, H. C.; Lira, H. L.; Carvalho, L. H.; Ávila Jr, M. M.; Pontes, M. K. G. \& Araújo, I. S. Mat. Sci. and Eng. B, 112, p.175 (2004).

15. Barbosa, R. - "Efeito de sais quaternários de amônio na organofilização de uma argila bentonita nacional para o desenvolvimento de nanocompósitos de polietileno de alta densidade (PEAD)", Dissertação de Mestrado, Universidade Federal de Campina Grande, Paraíba, Brasil (2005).

16. Araújo, E. M.; Melo, T. J. A.; Oliveira, A. D.; Araújo, H. L. D.; Araújo, K. D. \& Barbosa, R. - Polímeros: Ciência e Tecnologia, 16, p.38 (2006).

17. Araújo, E. M.; Barbosa, R.; Rodrigues, A.W.B. \& Ito, E. N. - Mat. Sci. and Eng. A, 445-446, p.141 (2007).

18. Cho, J. W. \& PAUL, D. R. - Polymer, 42, p.1083 (2001).

19. Sinha Ray, S. \& Okamoto, M. - Progress in Polymer Science, 28, p.1539 (2003).

20. Paz, R. A. - "Efeito do peso molecular da poliamida 6 no desenvolvimento de nanocompósitos", Dissertação de Mestrado, Universidade Federal de Campina Grande, Paraíba, Brasil (2008).

21. Kohan, I. M. - "Nylon Plastics Hanbook", Hanser Publishers, Munich (1995).

Enviado: 20/05/09

Reenviado: 04/07/09

Aceito: $27 / 07 / 09$ 